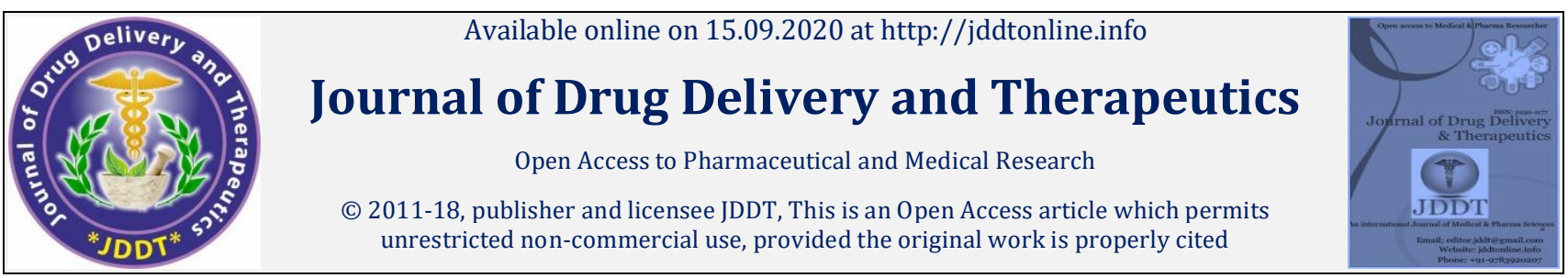

Open $\odot$ Access

Research Article

\title{
Evaluating the effect of the porous and non-porous colloidal silicon dioxide as a stabilizer on amorphous solid dispersion
}

\author{
Smruti P. Chaudhari, Mittal Bhadiyadra, Rutesh H. Dave* \\ Arnold and Marie Schwartz College of Pharmacy and Health Sciences, Division of Pharmaceutical Sciences, Long Island University, Brooklyn, \\ New York, 11201, USA
}

\begin{abstract}
Advancement in the discovery of drugs has led to many highly lipophilic compounds with very low water solubility. Amorphous solid dispersion is one of the emerging technologies to increase the solubility of these drugs. The stability of these systems is critical since the high energy system tends to recrystallize, which negates the benefits of these systems. In this paper, we are evaluating the use of colloidal silicon dioxide as a potential stabilizer to stabilize the amorphous solid dispersions. Two types of colloidal silicon dioxide are used: porous colloidal silicon dioxide -Syloid $244 \mathrm{Fp}$ and nonporous fumed silica - Aerosil 200. These silicon dioxides have a high surface area. Two methods of incorporation are used to incorporate silicon dioxide into the solid dispersion. The spray drying method is used to make amorphous solid dispersion. It was found that porous silicon dioxide is better to increase stability as well as increasing dissolution rate and \% release of the drug. The addition of silicon dioxide internally to the dispersion increases the dissolution rate, and the addition of silicon dioxide externally increases the stability of the solid dispersion.
\end{abstract}

Keywords: colloidal silicon dioxide, stabilizer, amorphous solid dispersion, low water solubility

Article Info: Received 19 July 2020; $\quad$ Review Completed 26 August 2020; $\quad$ Accepted 06 Sep 2020; $\quad$ Available online 15 Sep 2020 Cite this article as:

Chaudhari SP, Bhadiyadra M, Dave RH, Evaluating the effect of the porous and non-porous colloidal silicon dioxide as a stabilizer on amorphous solid dispersion, Journal of Drug Delivery and Therapeutics. 2020; 10(5):255-263 http://dx.doi.org/10.22270/jddt.v10i5.4323

*Address for Correspondence:

Rutesh H. Dave, Arnold and Marie Schwartz College of Pharmacy and Health Sciences, Division of Pharmaceutical Sciences, Long Island University, Brooklyn, New York, 11201, USA

\section{INTRODUCTION}

Solid dispersion is widely used in this decade to increase the solubility of poorly water-soluble BCS Class II drugs 1-3. These drugs have high permeability and thus the only barrier to increase bioavailability is the solubility of the drugs. As per the Noyes- Whitney equation, if we increase the solubility of the drug it will lead to increased absorption of the drugs 4 . In solid dispersion, the crystalline drug is converted into amorphous form thus increasing the solubility of the drug. Typically, solid dispersion consists of a hydrophilic drug and a hydrophobic polymer.

The crystalline form of the drug is thermodynamically stable. As we convert the drug in an amorphous state, it has very high energy and high mobility and thus thermodynamically less stable. Therefore, solid dispersions tend to recrystallize, this affects the drug solubility and dissolution profile of the drug5. The hydrophilic polymer also helps in increasing the stability of the amorphous drug to a certain extent.
Several methods are available for preparation of solid dispersions like hot melt extrusion (HME) ${ }^{6}$, fusion method 7 , supercritical fluid precipitation ${ }^{8}$, mechanical milling ${ }^{9}$, freeze drying 10 , and spray drying 11 . Out of these methods, HME and spray drying are widely used due to scalability aspects. However, HME involves use of high temperature and hence not suitable for low melting point drugs. In this research, we have used spray drying to prepare solid dispersion.

The addition of stabilizers in the solid dispersion can lead to a reduction in the energy and increase the stability of the $\mathrm{SD}^{12}, 13$. Many excipients have been used in literature as a stabilizer. In this research, we have used colloidal silicon dioxide as the stabilizer for the SD. Two different types of colloidal silicon dioxide are used: porous silica - Syliod 244 Fp and nonporous fumed silica Aerosil. Two different methods are used to incorporate this silica into solid dispersion.

In the first method, a stabilizer is added to the organic solution of the drug and polymer and subsequently spraydried and stored. In the second method, the stabilizer is mixed with the prepared solid dispersion physically and 
then stored. The addition of colloidal silicon dioxide also increases the powder flow and thus making the handling of solid dispersion easy in downstream processing.

In this paper, Fenofibrate (FENO) is used as a model drug to study the effect of incorporating colloidal silicon dioxide in the solid dispersion. Fenofibrate has very low water solubility and high permeability. The tg of the FENO is $21.3^{\circ} \mathrm{C}$. This model drug was chosen as it tends to spontaneous recrystallization.

\section{MATERIALS AND METHODS}

\section{Materials}

Feno was obtained from Sigma Aldrich. HPMC E5 (Methocel E5 LV) was procured from Dow chemicals. Syloid 244FP was procured from Grace and Aerosil was obtained from Evonik. The reagent like ethanol 190 proof was obtained from Sigma Aldrich.

\section{Preparation of solid dispersion.}

Solid dispersion was prepared by using Buchi Mini spray dryer B-290 (Buchi Laboretchnik AG, Flawil, Switzerland) connected to inert loop B-295. The drug was dissolved in water, and the polymer was dissolved in water. Both the solution was mixed to result in ethanol and water hydroalcoholic mixture (70:30). Silicon dioxide was dispersed in the formed solution. The feed concentration of $3.2 \%$ was used for spraying at a rate of $15 \mathrm{ml} / \mathrm{min}$ at $120 \mathrm{C}$ inlet temperature. The spray-dried dispersion obtained was then stored in a tightly closed vial at room temperature in desiccators.

\section{Characterization of Solid Dispersions:}

\section{Modulated Differential Scanning Calorimetry}

Modulated differential scanning calorimetry was performed using Q200 (TA Instruments, USA) equipped with a cooling system. Nitrogen was used as a purge gas with a flow rate of $50 \mathrm{ml} / \mathrm{min}$. 3-5 mg of sample was weighed into pinhole aluminum cell and hermetically sealed. Samples were heated up from $20-150^{\circ} \mathrm{C}$ at the heating rate of $5^{\circ} \mathrm{C} / \mathrm{min}$ with modulation of $1.59^{\circ} \mathrm{C}$ every 60 seconds.

The Heat-cool-heat cycle was used to analyze the physical mixtures. A single heat cycle was used for heating of the spray-dried solid dispersion samples.

\section{Fourier Transform Infrared Spectroscopy.}

The MAGNA-IR-60 Spectrophotometer (Nicolet Instrument Corp, Madison WI) was used to obtain Fourier Transformed Infrared spectroscopy (FTIR). The small quantity of all samples was triturated with pure potassium Bromide (KBr) and compressed to for the thin semitransparent film. The film was scanned for 400 to $4000 \mathrm{~cm}-1$ wavenumbers. A total of 64 scans were collected for each sample.

\section{Dissolution Testing}

Dissolution testing was carried using UPS Apparatus II. The deionized water (DI) and DI water with $1 \%$ SLS were used as testing media. The dispersion equivalent to $25 \mathrm{mg}$ of FENO was weighed and added to the $1000 \mathrm{ml}$ media. The media was maintained at $37{ }^{\circ} \mathrm{C}$ with a paddle speed of $75 \mathrm{RPM}$. The $5 \mathrm{ml}$ of sample is withdrawn after $5,10,15,30,45,60$ minutes of dissolution. Each sample is filtered using a 0.45 $\mu \mathrm{m}$ PTFE syringe filter and analyzed using a UV spectrophotometer at $288 \mathrm{~nm}$ wavelength.

\section{Stability Studies}

All the spray-dried dispersion was subjected to $40^{\circ} \mathrm{C}$ and $75 \%$ relative humidity (RH). Samples were pulled after 2,4 , and 6 weeks. After removal, the samples were thoroughly dried in silica gel $(0 \% \mathrm{RH})$ desiccators for 48 hours. The samples were then stored in a tightly sealed container in $0 \%$ $\mathrm{RH}$ desiccators for further analysis.

\section{RESULTS AND DISCUSSION}

\section{Solid dispersion}

Solid dispersion was prepared using a spray drying technique. The dispersion was prepared using a 1:5 Drug to polymer ratio. The silicon dioxide was added to the dispersion in two different ways. The colloidal silicon dioxide was added to the solution of drug and polymer and spray dried using the same parameter as the one without colloidal silicon dioxide. This solid dispersion is named as an internal solid dispersion. Another way of adding solid dispersion with colloidal silicon dioxide is by physically mixing the silicon dioxide to the solid dispersion. These solid dispersions are named as an external solid dispersion. Table 1 gives the list of the solid dispersion prepared.

Table 1: Solid dispersion composition

\begin{tabular}{|c|c|c|}
\hline $\begin{array}{c}\text { Solid } \\
\text { dispersion }\end{array}$ & Composition & Ratio \\
\hline SD1 & FENO:Methocel E5 & $1: 5$ \\
\hline ISD 1 & FENO:Methocel E5:Syloid 244FP & $1: 5: 1$ \\
\hline ISD 2 & FENO:Methocel E5:Syloid 244FP & $1: 5: 2$ \\
\hline ISD 3 & FENO:Methocel E5:Aerosil 200 & $1: 5: 1$ \\
\hline ISD 4 & FENO:Methocel E5:Aerosil 200 & $1: 5: 2$ \\
\hline ESD 1 & FENO:Methocel E5:Syloid 244FP & $1: 5: 1$ \\
\hline ESD 2 & FENO:Methocel E5:Syloid 244FP & $1: 5: 2$ \\
\hline ESD 3 & FENO:Methocel E5:Aerosil 200 & $1: 5: 1$ \\
\hline ESD 4 & FENO:Methocel E5:Aerosil 200 & $1: 5: 2$ \\
\hline
\end{tabular}

\section{Dissolution}

The dissolution of Feno and its dispersion was carried out in deionized water and deionized water with $1 \%$ SLS. The fenofibrate has very low water solubility and hence FENO and its physical mixtures do not release in water. The samples were analyzed using an ultraviolet spectrophotometer. This analysis technique does not detect the concentration below. The limit of quantification of this spectrophotometer was $2 \mu \mathrm{g} / \mathrm{ml}$. The solubility of the Feno in $1 \%$ SLS is $337 \mu \mathrm{g} / \mathrm{ml}$. Hence, DI water represents the nonsink condition, and $1 \%$ SLS represents the sink condition. As per literature, FENO dispersion dissolution in nonsink media correlates better with in vivo absorption profile 14 .

The release profile of all the internal solid dispersion and external solid dispersion in distilled water and the presence of 1\% SLS is shown in Figures 1 and 2 respectively. The pure fenofibrate is insoluble in water and it can also be seen from figure 1. From the release profile, it is evident that the addition of colloidal silicon dioxide internally increases the rate of dissolution and it is concentration-dependent. The addition of colloidal silicon dioxide externally also enhances the dissolution rate. However, if colloidal silicon dioxide is added internally more pronounced effect is produced. The syloid 244FP shows faster release as compared to Aerosil when used internally as well as externally. The release profiles are similar in both the media used. 

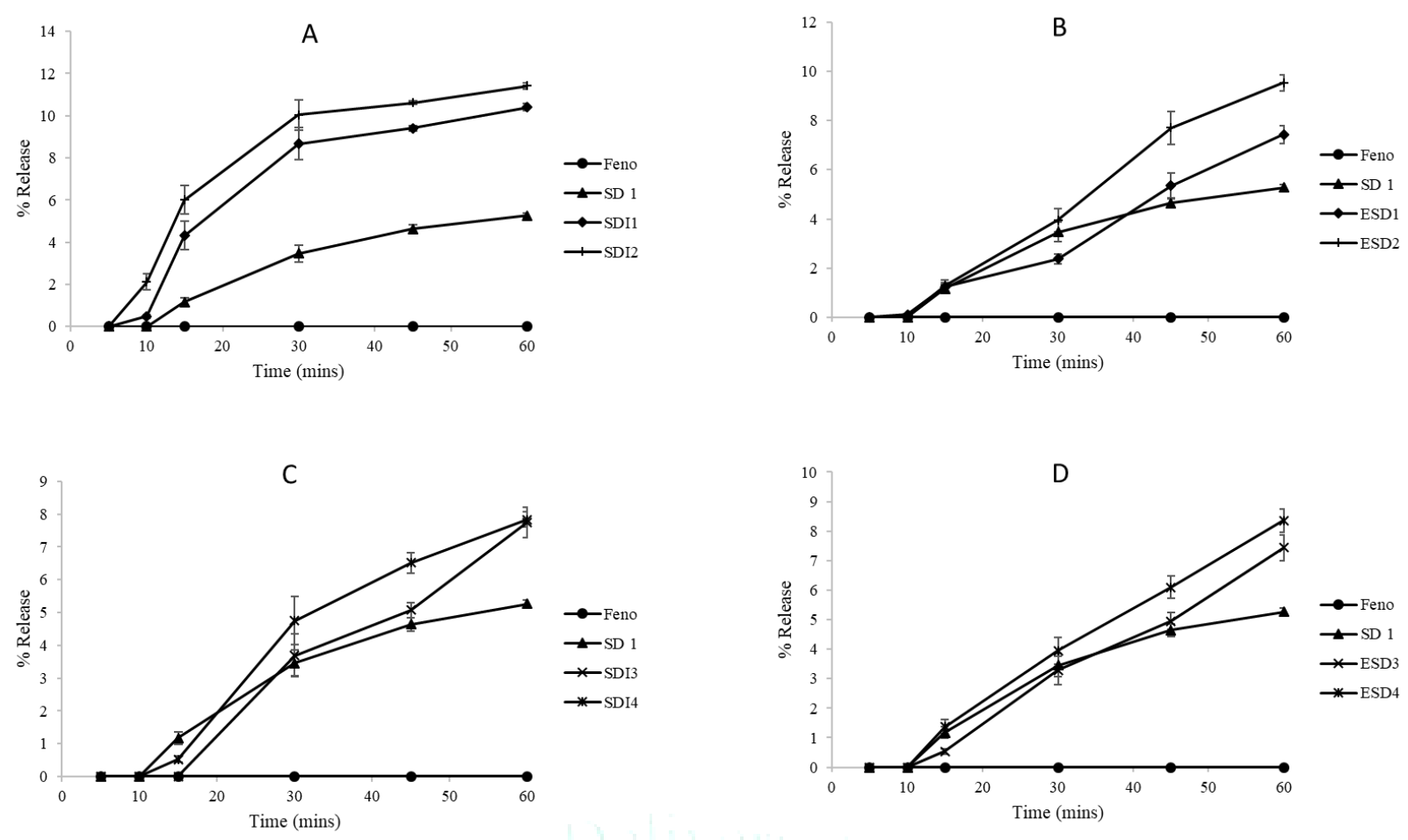

Figure 1: Dissolution of Fenofibrate, SD 1, SDI 1, SDI 2 SDI 3, SDI 3, ESD1, ESD2, ESD3, and ESD4 in DI water.

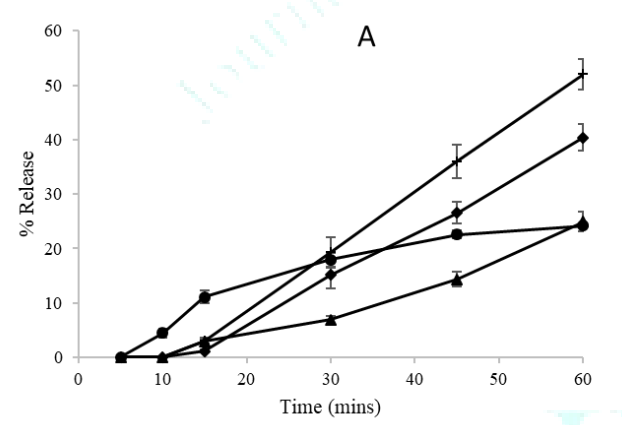

C

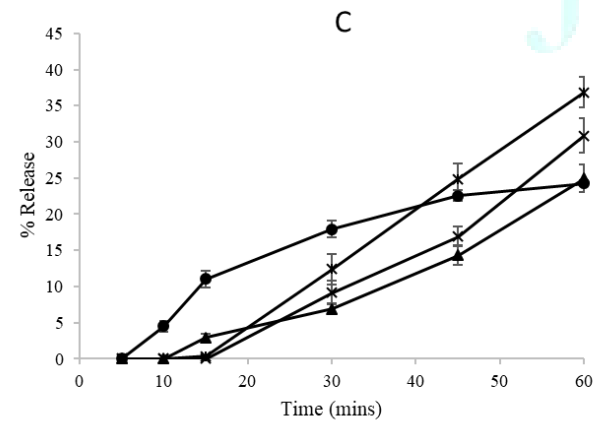

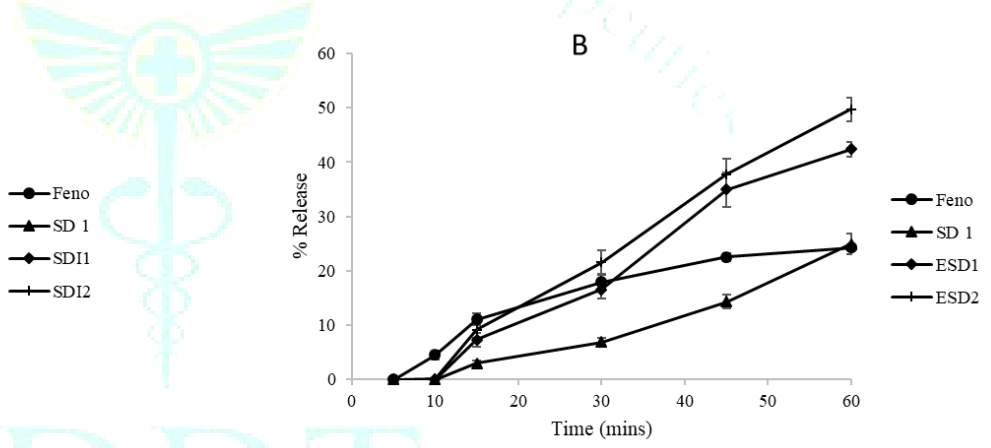

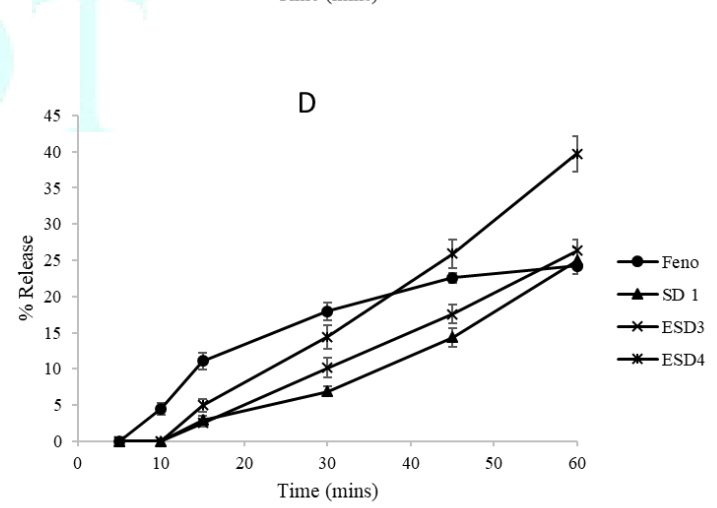

Figure 2: Dissolution of Fenofibrate, SD 1, SDI 1, SDI 2 SDI 3, SDI 3, ESD1, ESD2, ESD3, and ESD4 in DI water.

\section{Modulated Differential Scanning Calorimetry}

FENO is a crystalline drug converted into an amorphous state in solid dispersion. The amorphous state of the drug has high energy and therefore, it tends to convert into a stable crystalline form. The use of the polymers increases the stability of the amorphous solid dispersions. Figure 3 shows the DSC thermograms of the solid dispersions. All the dispersions have the absence of crystalline FENO peak, indicating the amorphous nature of the dispersions. Figure 4 shows the thermograms of stability samples. All the dispersions show the recrystallization of the FENO. In this study, the stability samples were stored at 40C and $75 \%$ humidity in the open vials. The open vials allow the humidity exchange. The water acts as the media and increases the mobility of the molecule resulting in the recrystallization. The polymer and the stabilizer are used to inhibit the recrystallization. Hence, it will increase the stability of the amorphous solid dispersion when stored in appropriate packaging with desiccants. The percent crystallinity of the 
Chaudhari et al

solid dispersions and its stability samples are calculated with the help of Eq1 15, 16 .

Crystallinity of Drug Substance $=\frac{\frac{A}{W t}}{H} \times 100 \%$ Eq 1

Where, $\mathrm{A}=$ area under the melting endotherm, $\mathrm{Wt}=$ amount of FENO in the solid dispersion and $\mathrm{H}=$ heat of fusion of pure crystalline FENO.

The DSC thermograms of the pure FENO and its dispersions are shown in figure 3. The thermograms of the stability sample of solid dispersion SD 1 is shown in figure 4. The thermograms of the internal and external solid dispersion and its stability samples are given in figure 5 and 6 respectively. It is seen in thermograms that on stability there is recrystallization of the FENO. Figure 7 shows the percent crystallinity of all the stability samples. It is seen that Solid
Journal of Drug Delivery \& Therapeutics. 2020; 10(5):255-263 dispersion is stable for 2 weeks and after 2 weeks it showed approximately $40 \%$ of crystallinity at 4 weeks. The addition of stabilizers increases stability and it increases as the amount of stabilizer is increased. The method of addition also has an impact on the stability of the dispersion. When Syloid 244 Fp is used as a stabilizer, the addition of stabilizer externally shows the lower crystallinity i.e. increased stability. Whereas for Aerosil no significant difference is seen in internal and external solid dispersion. This is attributed to the nonporous nature of the Aerosil. The Syloid 244FP has the porous nature and hence it is more effective to increase the release rate and when added externally it creates physical separation which avoids two molecules of FENO to come together to recrystallize.

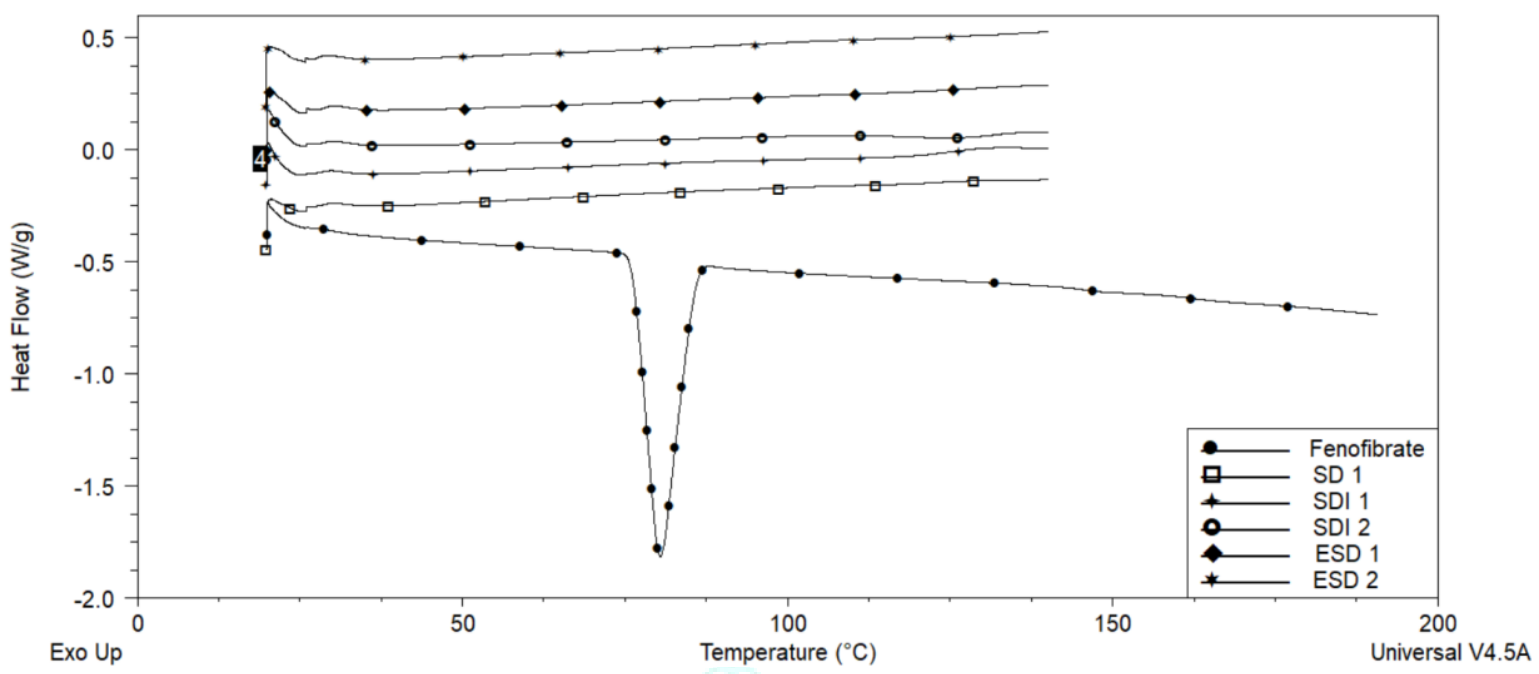

A

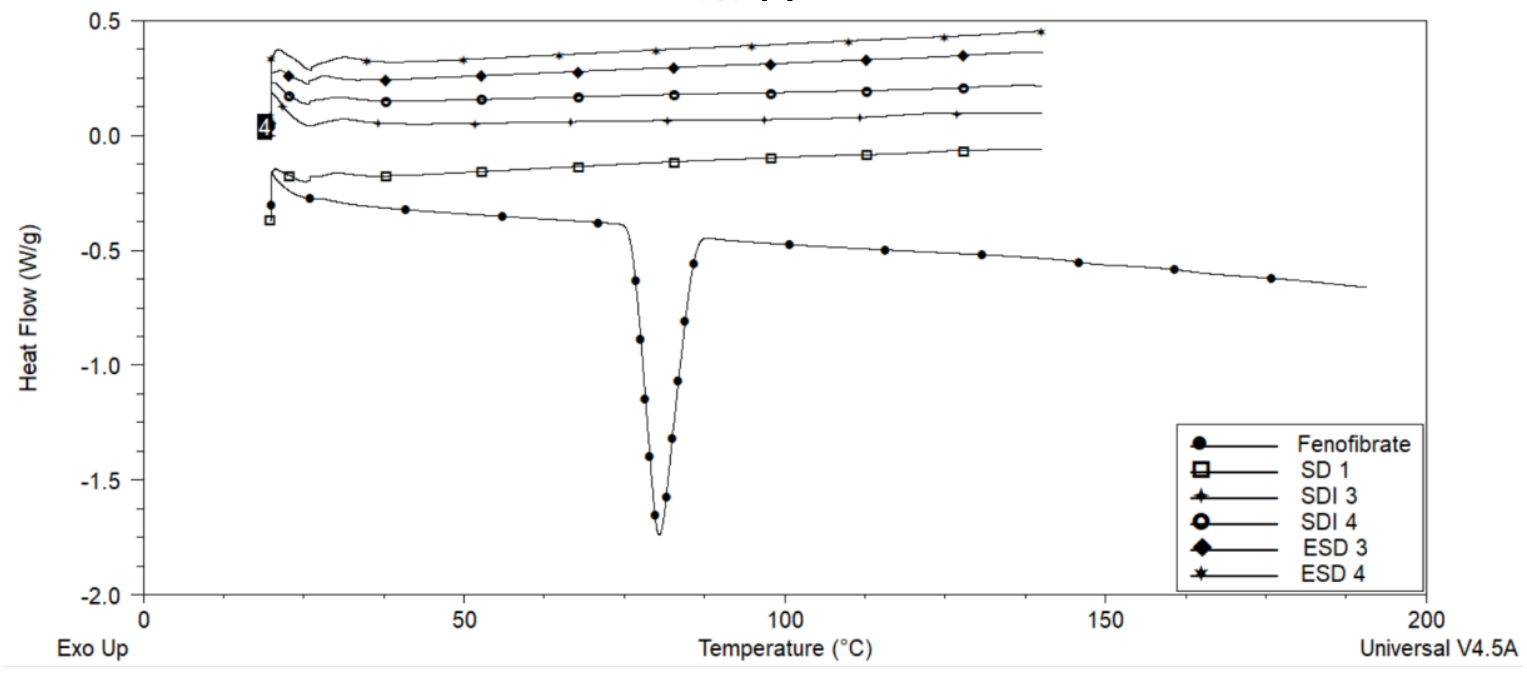

B

Figure 3: A: DSC thermograms of the pure FENO and syloid 244 FP solid dispersions, B: DSC DSC thermograms of the pure FENO and Aerosil solid dispersions 


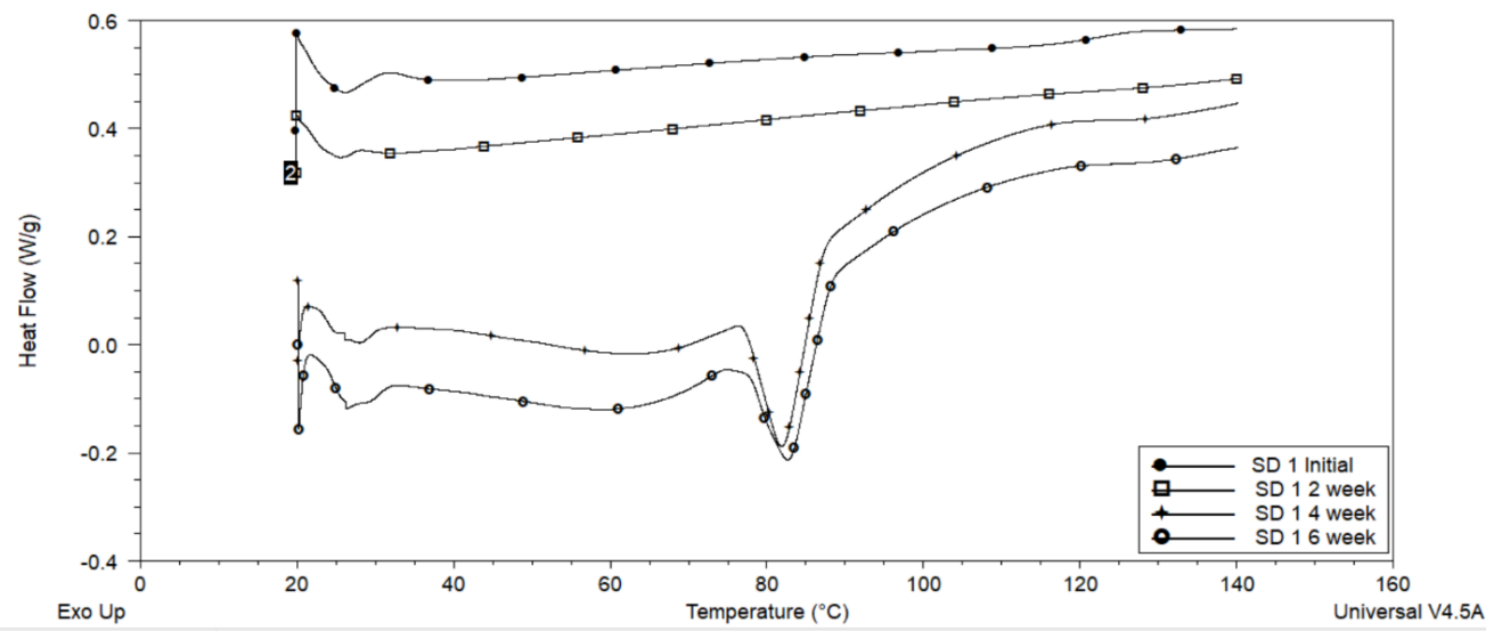

Figure 4: DSC thermograms of the soldi dispersion SD 1 and its stability samples
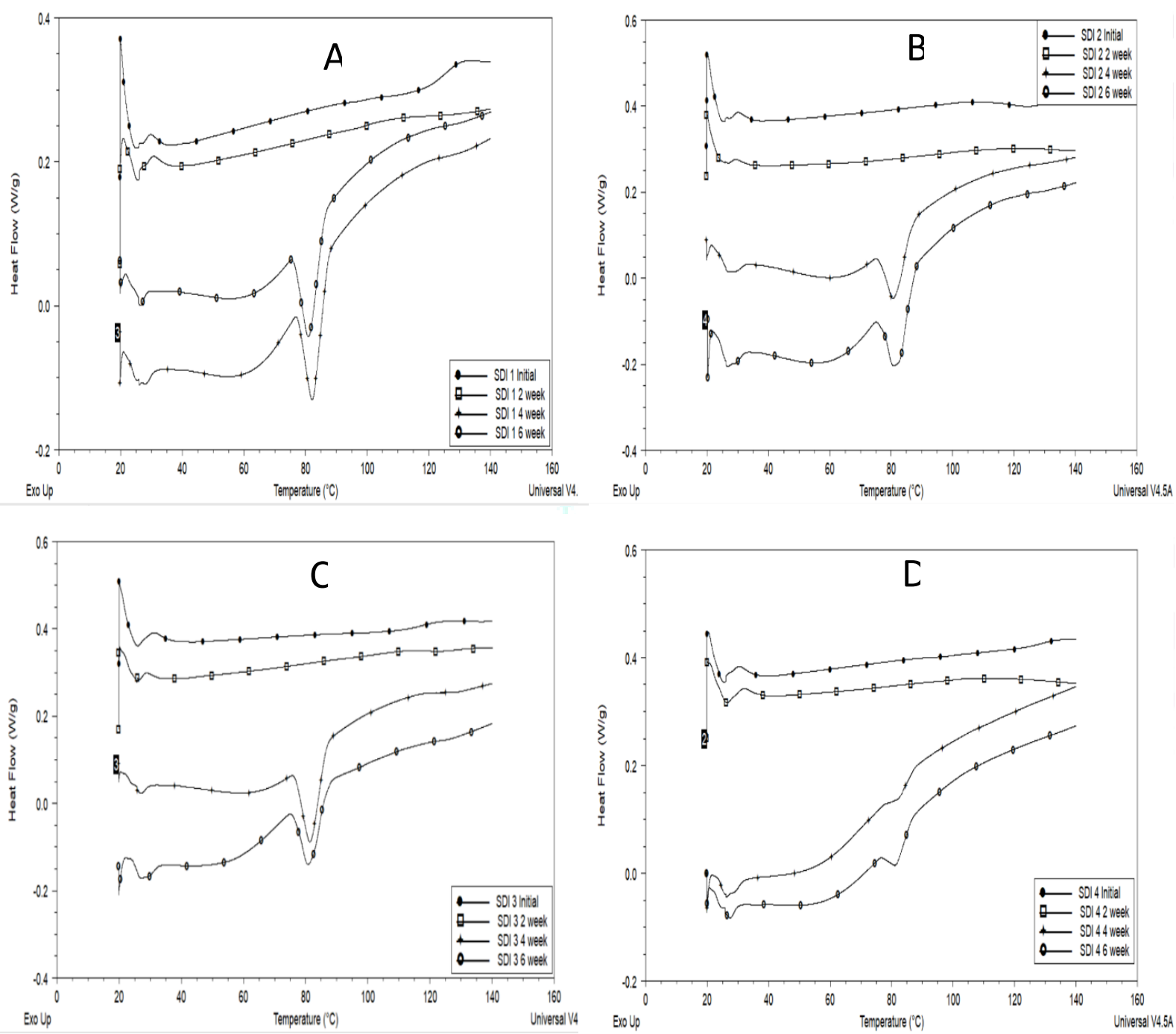

Figure 5: DSC thermograms of the internal solid dispersion and its dispersions 

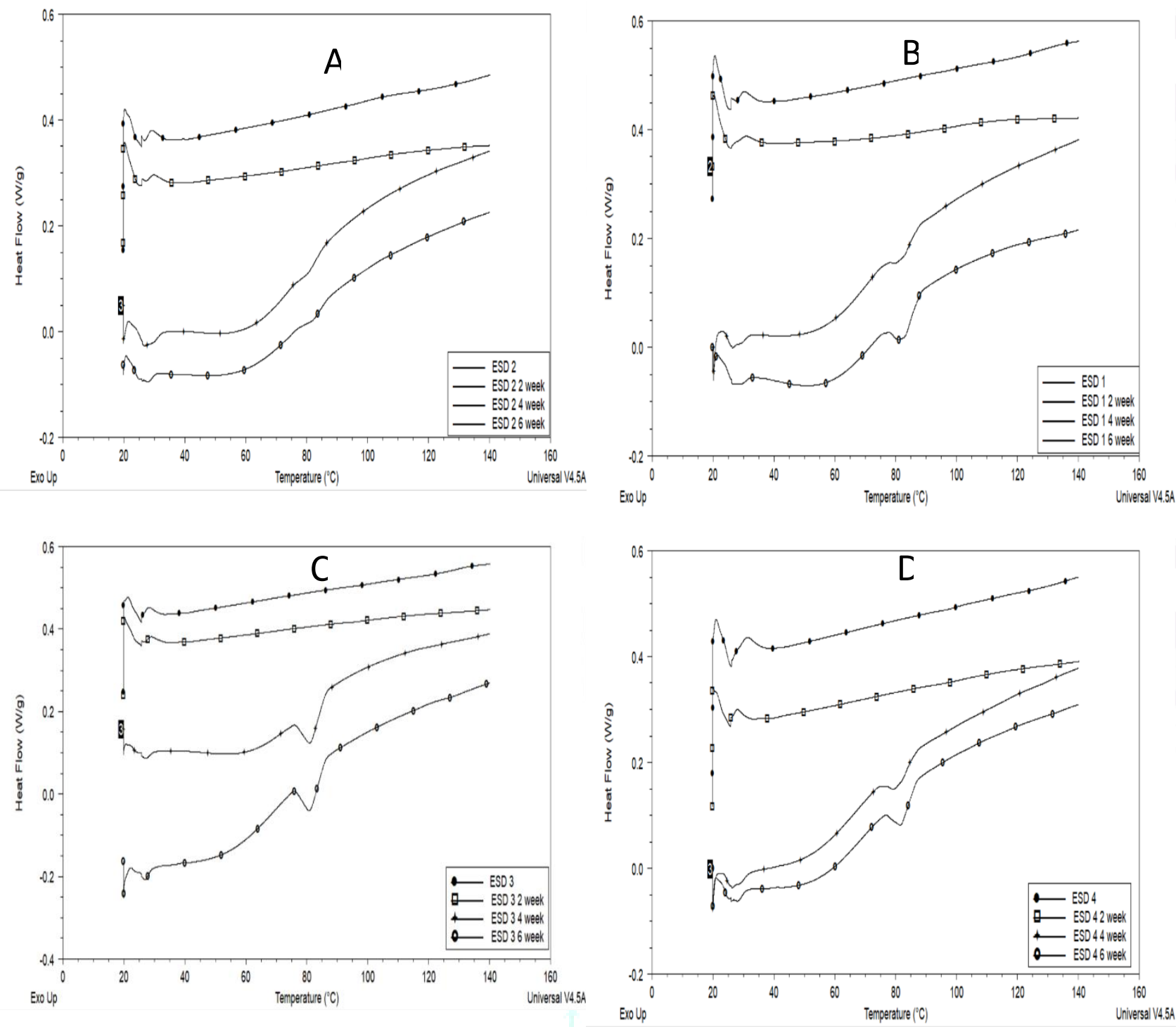

Figure 6: DSC thermograms of the External solid dispersion and its dispersions

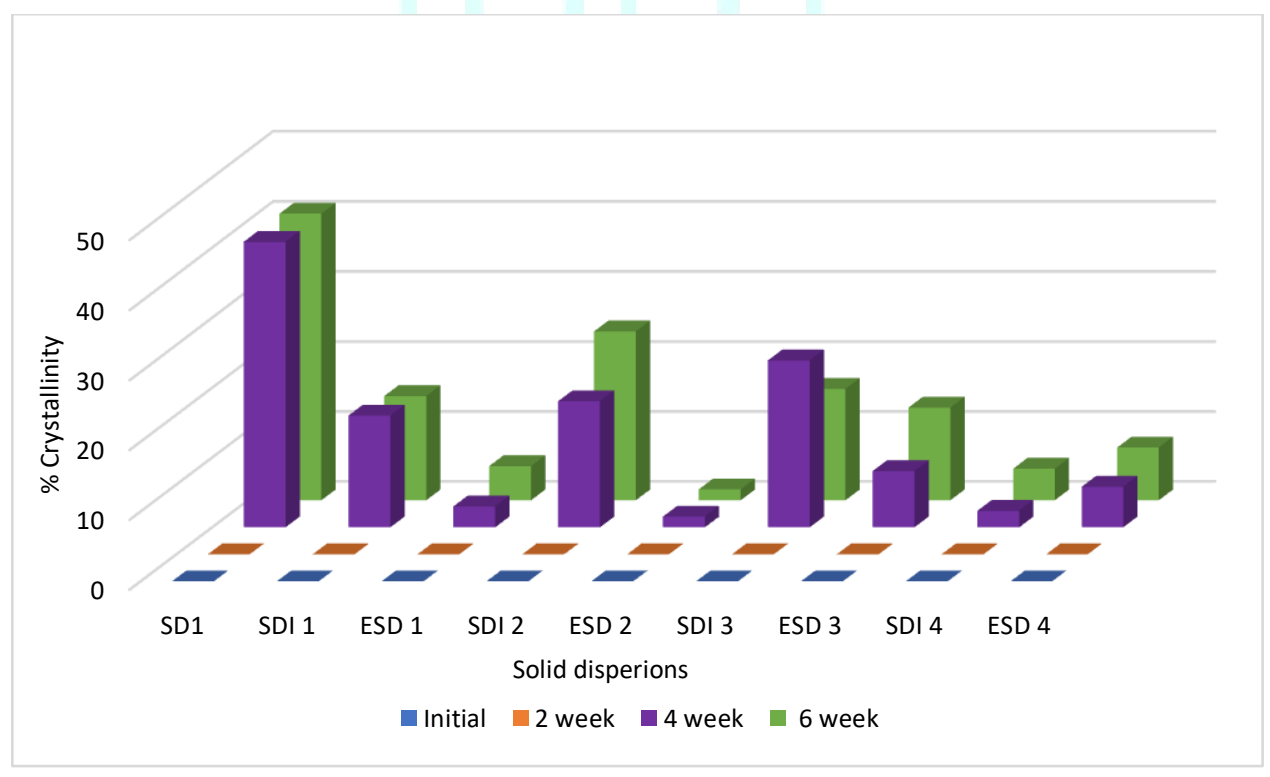

Figure 7: \% crystallinity of solid dispersions 


\section{Fourier Transformed Infrared Spectroscopy}

FTIR was performed to determine Drug-polymer interactions. The interactions can be in the form of hydrogen bonding or van der Waal attraction forces. It helps stabilize the system ${ }^{17}$.

Crystalline fenofibrate has four functional groups which can act as a hydrogen acceptor, two hydroxyl groups ( $\mathrm{OH}$ group), and two oxygen atoms of the carbonyl group $(\mathrm{C}=0)$ but it lacks a proton donor. It is being reported that hydrogen bonds formed between drugs and polymers help to stabilize the amorphous system.

Figure 8 shows the FTIR spectra of Syloid 244FP solid dispersion initial and at 6 weeks. Figure 9 shows the FTIR spectra of Aerosil solid dispersion initial and at 6 week. Fenofibrate in crystalline form shows two peaks of carbonyl carbon stretching at $1729 \mathrm{~cm}-1$ and $1652 \mathrm{~cm}-1$. The less resolved and broader band peaks shapes in FTIR spectra indicate the presence of amorphous fenofibrate ${ }^{18}$. Shifting of peaks in IR spectra determines the strength of hydrogen bonds.

IR spectra of pure FENO are shown in figure 8. It shows peaks at $1729 \mathrm{~cm}-1$ and at $1652 \mathrm{~cm}-1$. All solid dispersions show the shifting of peaks from $1729 \mathrm{~cm}-1$ and at $1652 \mathrm{~cm}$ 1. Solid dispersion without stabilizer (SD 4) shows a peak at $1733 \mathrm{~cm}-1$ and $1657 \mathrm{~cm}-1$. Solid dispersion with syloid as a stabilizer (SDI 1 and SDI 2) shows a peak at $1734 \mathrm{~cm}-1$ and $1657 \mathrm{~cm}-1,1736 \mathrm{~cm}-1$, and at $1657 \mathrm{~cm}-1$ respectively. Whereas solid dispersion with aerosil as stabilizer shows a peak at $1731 \mathrm{~cm}-1$ and $1656 \mathrm{~cm}-1,1736 \mathrm{~cm}-1$, and at 1656 cm-1 respectively. The shift of peaks toward higher wavelengths in solid dispersion with stabilizer indicates the formation of stronger hydrogen bonds as compared to solid dispersion without stabilizers.

On stability, the SD 1 shows peaks similar to pure fenofibrate indicating the conversion of amorphous FENO to the crystalline state. IR spectra of 6-week samples for SDI 1 and SDI 2 show the peak of carbonyl stretching at $1733 \mathrm{~cm}-1$ and $1653 \mathrm{~cm}-1,1736 \mathrm{~cm}-1$, and at $1653 \mathrm{~cm}-1$ respectively. Whereas for IR spectra for 6-week samples of solid dispersion SDI 3 and SDI 4 shows peaks at $1730 \mathrm{~cm}-1$ and $1652 \mathrm{~cm}-1,1735 \mathrm{~cm}-1$, and at $1654 \mathrm{~cm}-1$ respectively. This indicates that solid dispersion with both stabilizers helps to stabilize amorphous solid dispersion as it shows no spectral shift on storage, indicating no conversion of amorphous form into crystalline fenofibrate.

IR spectra of external solid dispersion, all of them show less shift as compared to SD 1. This data supports the fact that SD 1 shows higher crystallinity as compared to solid dispersion with stabilizers. In some cases, it is demonstrated that drugpolymer interactions are important to stabilize solid dispersion. Some researchers argued that the antiplasticizing effect of polymer plays an important role in stabilizing amorphous solid dispersion. From the results, it can be said that the type of stabilizer as well as the amount of stabilizer affects the amorphous characteristic of solid dispersions.

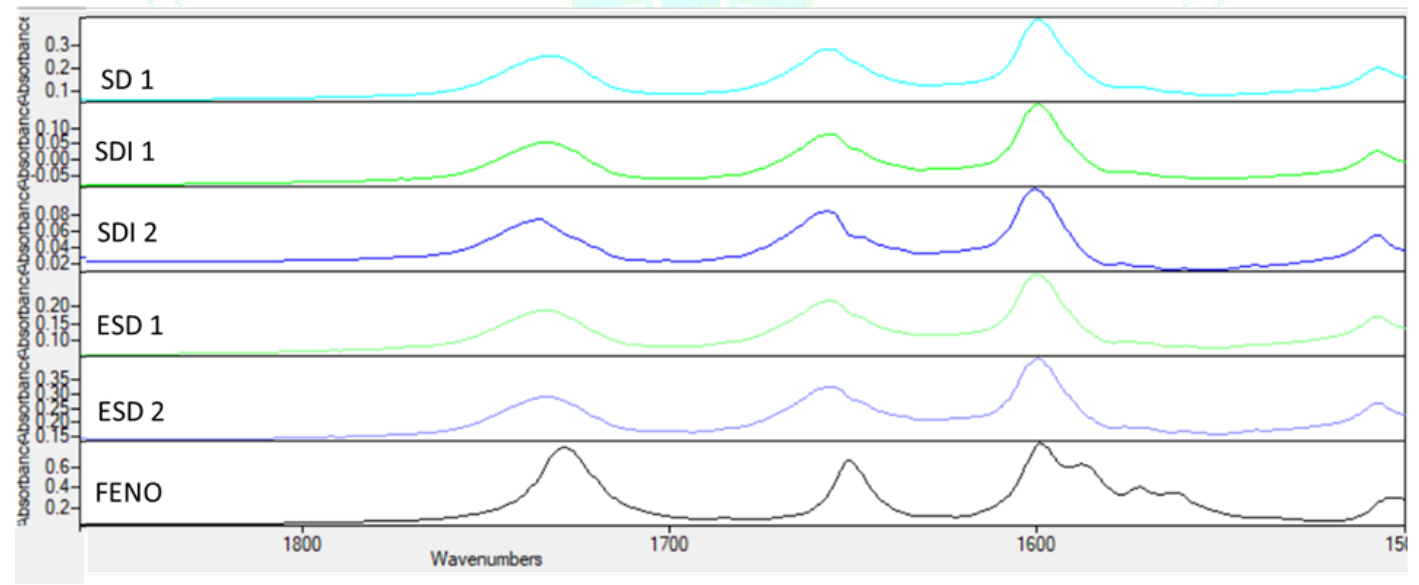

A

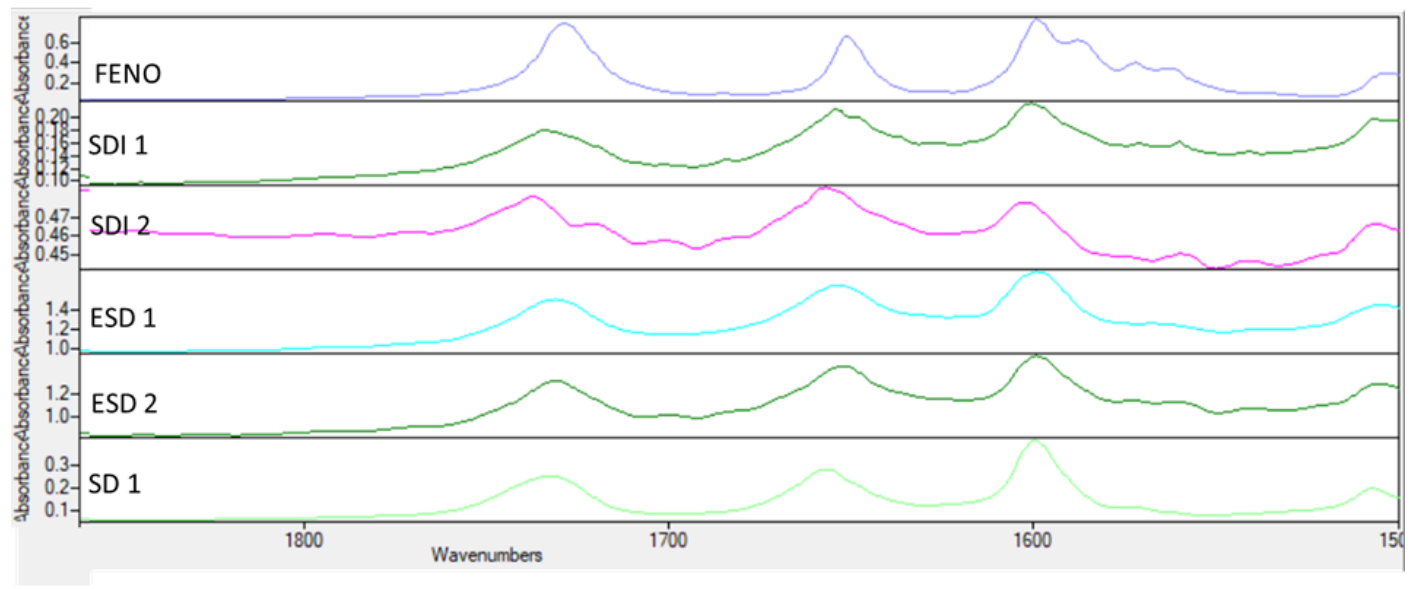

B

Figure 8: FTIR Spectra of Syloid 244 Fp solid dispersion A: Initial and B: 6 week stability sample 


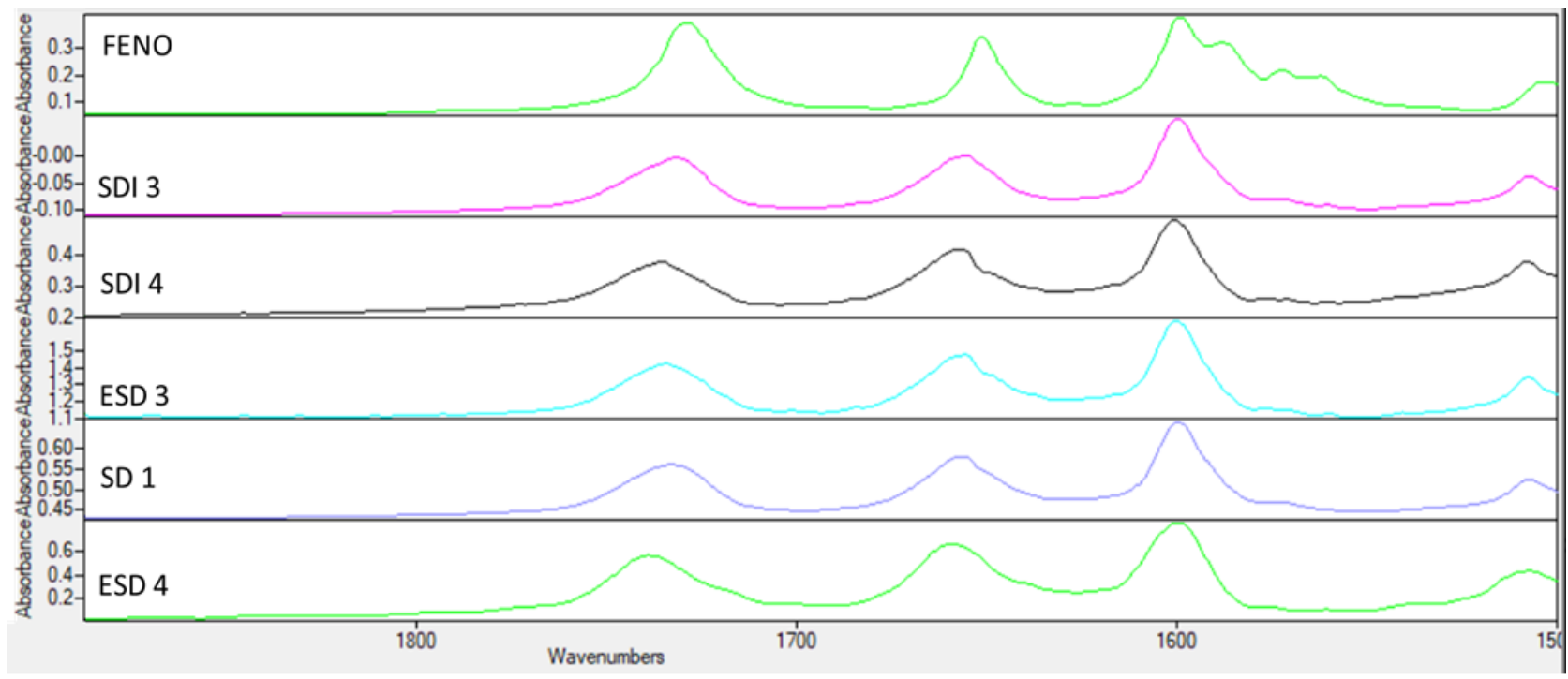

A

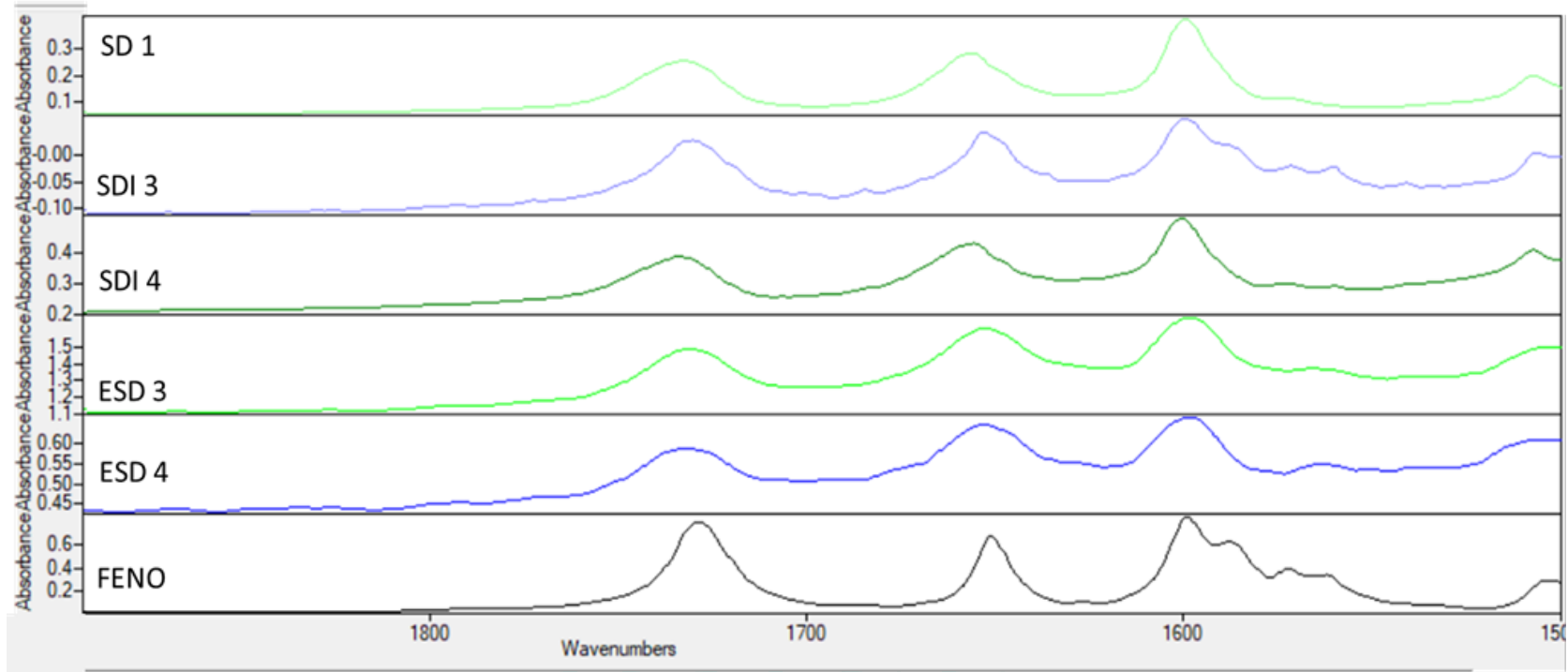

B

Figure 9: FTIR Spectra of Aerosil solid dispersion A: Initial and B: 6 week stability sample

\section{CONCLUSION:}

This study was performed to evaluate the colloidal silicon dioxide as a stabilizer for FENO solid dispersions. Two different grades of colloidal silica were studied, SYLOID 244FP-porous and Aerosil 200-nonporous. It was concluded that both the porous and nonporous colloidal silicon dioxide enhances the release rate of solid dispersions. It was concentration dependent. The porous colloidal silicon dioxide shows better results as compared to nonporous colloidal silicon dioxide. The dispersion was subjected to elevated temperature and humidity. As expected, FENO recrystallizes out and colloidal silicon dioxide enhances the stability by hindering the conversion of FENO from amorphous form to the crystalline state. The porous colloidal silicon dioxide is seen to have better stability as compared to nonporous colloidal silicon dioxide. The internal colloidal silicon dioxide enhances the dissolution rate and release, but external colloidal silicon dioxide improves the stability. It is concluded that colloidal silicon dioxide ineffective to enhance dissolution rate and stability.
The combination of internal colloidal silicon dioxide and external colloidal silicon dioxide needs to be used to maximize the benefits.

\section{Conflict of interest}

Author reports no conflict of interest.

\section{REFERENCES}

1. Lipinski CA. Drug-like properties and the causes of poor solubility and poor permeability. J Pharmacol Toxicol Methods. 2000; 44(1):235-49.

2. Gribbon P, Andreas S. High-throughput drug discovery: What can we expect from HTS? Drug Discovery Today. 2005; 10(1):17-22.

3. Singh A, Worku ZA, Van den Mooter G. Oral formulation strategies to improve solubility of poorly water-soluble drugs. Expert Opinion on Drug Delivery. 2011; 8(10):1361-78.

4. Guzmán HR, Tawa M, Zhang Z, Ratanabanangkoon P, Shaw $P$, Gardner CR, et al. Combined use of crystalline salt forms and precipitation inhibitors to improve oral absorption of celecoxib from solid oral formulations. J Pharm Sci. 2007; 96(10):2686702. 
5. Patil VS, Dziubla TD, Kalika DS. Static and dynamic properties of biodegradable poly (antioxidant $\beta$-amino ester) networks based on incorporation of curcumin multiacrylate. Polymer. 2015; 75:88-96.

6. Lakshman JP, Cao Y, Kowalski J, Serajuddin ATM. Application of Melt Extrusion in the Development of a Physically and Chemically Stable High-Energy Amorphous Solid Dispersion of a Poorly Water-Soluble Drug. Molecular Pharmaceutics. 2008; 5(6):994-1002.

7. DiNunzio JC, Brough C, Hughey JR, Miller DA, Williams Iii RO, McGinity JW. Fusion production of solid dispersions containing a heat-sensitive active ingredient by hot melt extrusion and Kinetisol® dispersing. Eur J Pharm Biopharm. 2010; 74(2):34051.

8. Bouchard A, Jovanović N, Hofland GW, Jiskoot W, Mendes E, Crommelin DJA, et al. Supercritical fluid drying of carbohydrates: Selection of suitable excipients and process conditions. Eur J Pharm Biopharm. 2008; 68(3):781-94.

9. Peltonen L, Hirvonen J. Pharmaceutical nanocrystals by nanomilling: critical process parameters, particle fracturing and stabilization methods. J Pharm Pharmacol. 2010; 62(11):156979.

10. Yang W, Tam J, Miller DA, Zhou J, McConville JT, Johnston KP, et al. High bioavailability from nebulized itraconazole nanoparticle dispersions with biocompatible stabilizers. Int J Pharm. 2008; 361(1-2):177-88.

11. Patel AD, Agrawal A, Dave RH. Development of polyvinylpyrrolidone-based spray-dried solid dispersions using response surface model and ensemble artificial neural network. J Pharm Sci. 2013; 102(6):1847-58.
12. Chaudhari SP, Gupte A. Mesoporous silica as a carrier for amorphous solid dispersion. arXiv preprint arXiv:170700036. 2017.

13. Patel VI, Dave RH. Evaluation of Colloidal Solid Dispersions: Physiochemical Considerations and In Vitro Release Profile. AAPS PharmSciTech. 2013; 14(2):620-8.

14. Chaudhari SP, Dave RH. Evaluating the Effects of Different Molecular Weights of Polymers in Stabilizing Supersaturated Drug Solutions and Formulations Using Various Methodologies of the Model Drug: Fenofibrate. J Pharm Sci Pharmacol. 2015; 2(3):259-76.

15. Pan X, Julian T, Augsburger L. Quantitative measurement of indomethacin crystallinity in indomethacin-silica gel binary system using differential scanning calorimetry and X-ray powder diffractometry. AAPS PharmSciTech. 2006; 7(1):E72E8.

16. Pan X, Julian T, Augsburger L. Increasing the Dissolution Rate of a Low-Solubility Drug Through a Crystalline-Amorphous Transition: A Case Study with Indomethicin. Drug Dev Ind Pharm. 2008; 34(2):221-31.

17. Lynne S. Taylor GZ. Spectroscopic Characterization of Interactions Between PVP and Indomethacin in Amorphous Molecular Dispersions. Pharm Res. 1997; 14(12):1691-8.

18. Heinz A, Gordon KC, McGoverin CM, Rades T, Strachan CJ. Understanding the solid-state forms of fenofibrate - A spectroscopic and computational study. Eur J Pharm Biopharm. 2009; 71(1):100-8. 